\title{
ADAPTACIÓN DE LA DOCENCIA PRÁCTICA DE MATERIAS DE BIOQUÍMICA EN TIEMPOS DE LA PANDEMIA POR EL COVID-19
}

\author{
Vizoso-Vázquez, Angel'; Rodríguez-Torres Ana M. ${ }^{2}$; Freire-Picos, María A. ${ }^{3}$ \\ ${ }^{1}$ Universidade da Coruña, Facultad de Ciencias, Código ORCID 0000-0001-7310-2102 \\ ${ }^{2}$ Universidade da Coruña, Facultad de Ciencias, Código ORCID 0000-0002-8303-6038 \\ ${ }^{3}$ Universidade da Coruña, Facultad de Ciencias, Código ORCID 0000-0002-8130-5388
}

\section{RESUMEN}

En este trabajo se comentan los retos que nos vimos obligados a afrontar y las soluciones tomadas por el profesorado a fin de adaptar la docencia de prácticas de laboratorio a la situación de pandemia causada por el SARS-CoV2 (Covid-19). Esta situación, además de ser nueva, fue variando con el tiempo principalmente a causa de confinamientos y variaciones en las restricciones de movilidad. Por tanto, pasamos de momentos con docencia completamente virtual a situaciones con docencia híbrida y también con clases presenciales. Presentamos nuestra experiencia adaptando el sistema tradicional de docencia práctica con grupos de 16-18 alumnos en un laboratorio manejando instrumental y compartiendo espacios a una situación de docencia con oscilaciones entre presencial, virtual e híbrida.

PALABRAS CLAVE: Covid19, Prácticas de laboratorio, innovación docente, docencia virtual, docencia híbrida. 


\section{CITA RECOMENDADA:}

Vizoso Vázquez, Ángel; Rodríguez Torres Ana M.; Freire Picos, María A. (2021): Adaptación de la docencia práctica de materias de Bioquímica en tiempos de la pandemia por el Covid-19. En García Naya, J.A. (ed.) (2021). Contextos universitarios transformadores: a nova normalidade académica. Leccións aprendidas e retos de futuro. V Xornadas de Innovación Docente. Cufie. Universidade da Coruña. A Coruña (pág. 433-441).

DOI capítulo: https://doi.org/10.17979/spudc.9788497498180.433

DOl libro: https://doi.org/10.17979/spudc.9788497498180

\section{ABSTRACT}

This paper discusses the challenges that we were forced to face and the solutions taken by the teaching staff in order to adapt the teaching of laboratory practices to the pandemic situation caused by SARSCoV2 (Covid-19). This situation, in addition to being new, varied over time mainly due to confinements and variations in mobility restrictions. Therefore, we went from moments with completely virtual teaching to situations with hybrid teaching and also with face-to-face classes. We present our experience adapting the traditional system of practical teaching with groups of 16-18 students in a laboratory, handling instruments and sharing space to a teaching situation with oscillations between face-to-face, virtual and hybrid.

KEYWORDS: Covid19, laboratory practices, teaching innovation, virtual-teaching, hybrid-teaching. 


\section{INTRODUCCIÓN}

Las carreras de ciencias, como en el caso que nos ocupa, la Biología, tienen una docencia en la que las prácticas de laboratorio resultan esenciales tanto para la mejor comprensión de contenidos como para el desarrollo de las competencias necesarias para su futuro desempeño profesional. En la Facultad de Ciencias de la UDC las prácticas de laboratorio son obligatorias para los estudiantes. Esta circunstancia se extiende al alumnado de las asignaturas de bioquímica en las que nos centraremos en este trabajo: las Bioquímicas I y II (BQ-I y $B Q-I I)$ y la Bioquímica y Biología Molecular (BBM) del Grado en Biología.

En marzo del año 2020 se declaró en España el estado de alarma que supuso el confinamiento de toda la población, excepto los trabajadores considerados esenciales. En esta situación, las universidades volcaron toda su docencia presencial a la modalidad virtual y esta situación no solo afectaba a España sino también a muchos otros países (Zhu and Liu, 2020). Esto supuso un gran reto para el profesorado y los estudiantes (Nuere, and De Miguel, 2020) que además requería de una formación adicional para el manejo de muchas herramientas de teleformación (Picón et al., 2020).

En el caso de la Universidade da Coruña, UDC, el profesorado recibió clases de teleformación en sesiones virtuales con expertos del servicio de tecnologías de la información y las comunicaciones y del personal CUFIE, ambos de la UDC, que además estuvo a disposición de las consultas del profesorado para resolver cualquier duda o incidencia. Con esta preparación preliminar, estudiantes y profesorado nos manejamos sobre todo al principio, mediante ensayo-error y también ayudándonos en el momento que surgían problemas con las plataformas durante sesiones de clase. Esta experiencia ayudó mucho al diseño de sesiones virtuales y grabación de videos que en el caso del trabajo que se presenta fueron esenciales para el diseño de prácticas virtuales. 


\section{DESCRIPCIÓN Y RESULTADOS DE LA EXPERIENCIA}

Siguiendo un orden cronológico que se relaciona en la tabla 1, la primera asignatura que se vio afectada por la pandemia fue la Bioquímica -I del primer curso del Grado en Biología. Estas prácticas se imparten a mediados del segundo cuatrimestre y fueron completamente virtuales a causa del confinamiento.

\begin{tabular}{|c|c|c|c|c|}
\hline \multirow[b]{2}{*}{ Período del curso } & \multirow[b]{2}{*}{ Asignatura } & \multicolumn{2}{|c|}{ Modalidad/grupos } & \multirow[b]{2}{*}{ Ola Covid19 } \\
\hline & & Virtual & Presencial* & \\
\hline 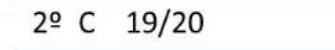 & $B Q-1$ & Sí todos & No & 1 a \\
\hline $1^{\text {er }}$ C $20 / 21$ & $B Q-I I$ & No & Sí todos & 2 \\
\hline 20 C $20 / 21$ & BBM & 4 grupos $/ 7 \rightarrow$ & 3 grupos $/ 7$ & 3ạ \\
\hline \multicolumn{2}{|c|}{$\begin{array}{l}\text { C: cuatrimestre } \\
{ }^{*} \text { con reducción de alumnos/sesión }\end{array}$} & \multicolumn{2}{|c|}{$\begin{array}{l}\text { Cambio en las restricciones } \\
\text { Gobierno autonómico }\end{array}$} & \\
\hline \multicolumn{2}{|l|}{ BQ-I: Bioquímica-I } & \multicolumn{3}{|c|}{ BBM: Bioquímica y Biología Molecular } \\
\hline
\end{tabular}

Tabla 1. Variaciones de la presencialidad de las prácticas de laboratorio según el estado de la pandemia.

En primer lugar, cabe destacar que se adaptaron estas prácticas reduciendo los contenidos a fin de no dar un exceso de información; además la adaptación fue coordinada con el profesorado de segundo curso con la idea de adaptar las prácticas del año siguiente (en la asignatura Bioquímica-II) para que se adquiriese experiencia en el manejo de instrumental en el laboratorio de prácticas.

Se elaboraron videos y explicaciones online de las prácticas además de tutorías con la profesora. Estos materiales estuvieron a disposición de los estudiantes en la plataforma Moodle de la asignatura (tabla 2). 


\begin{tabular}{|c|c|c|c|c|}
\hline \multirow[b]{2}{*}{ Período del curso } & \multirow[b]{2}{*}{ Asignatura } & \multicolumn{2}{|c|}{ Modalidad/grupos } & \multirow[b]{2}{*}{ Ola Covid19 } \\
\hline & & Virtual & Presencial & \\
\hline 2o C $20 / 21$ & $B Q-1$ & 7 grupos & No & $1 \underline{a}$ \\
\hline $\begin{array}{l}\text { Guión de } \\
\text { prácticas }\end{array}$ & \multicolumn{4}{|c|}{ Adaptado y simplificado, disponible en el Moodle. } \\
\hline $\begin{array}{l}\text { Explicaciones } \\
\text { online }\end{array}$ & \multicolumn{4}{|c|}{$\begin{array}{l}\text { A través de: Moodle videos, presentaciones. } \\
\text { Teams explicaciones y tutorías grupales e individuales. }\end{array}$} \\
\hline $\begin{array}{l}\text { Resolución de } \\
\text { ejercicios }\end{array}$ & \multicolumn{4}{|c|}{$\begin{array}{l}\text { A través de: Moodle: ejercicios resueltos y videos. } \\
\text { Teams: con videos explicaciones de los ejercicios }\end{array}$} \\
\hline $\begin{array}{l}\text { Examen } \\
\text { online }\end{array}$ & \multicolumn{4}{|c|}{ A través de Moodle } \\
\hline
\end{tabular}

Tabla 2. Adaptaciones y circunstancias durante el desarrollo de la docencia práctica en la asignatura Bioquímica I (BQ-I) durante el confinamiento de la primera Ola de Covid19.

Al iniciar el curso 2021 se estaba terminando la segunda pandemia y las primeras semanas la docencia en la Facultad de Ciencias fue completamente virtual para las clases teóricas y de grupos reducidos. Sin embargo, las prácticas fueron presenciales y se realizaron con los grupos duplicados para tener un menor número de estudiantes compartiendo espacio. Este fue el caso de la Bioquímica-II (tabla1). Además, se coordinaron los subgrupos creados con otras asignaturas del mismo curso favoreciendo así que en un turno (de mañana 0 tarde) los mismos alumnos asistiesen a las dos asignaturas consecutivamente reduciendo así sus desplazamientos a la Facultad de Ciencias.

El guion de prácticas de Bioquímica-ll se modificó con dos objetivos: añadir experimentos de Bioquímica-I no vistos el curso anterior por la adaptación a la pandemia y simplificar los contenidos acortando, por ejemplo, el número de muestras por práctica y combinando contenidos de dos prácticas de las que se impartían en años anteriores. En el Moodle de la asignatura además del guion de prácticas modificado, se incluyeron enlaces a vídeos explicativos de las técnicas. 


\begin{tabular}{lllll} 
& & \multicolumn{2}{c}{ Modalidad/grupos } & \\
\cline { 3 - 4 } Período del curso & Asignatura & Virtual & Presencial* & Ola Covid19 \\
\hline 2ㅇ C $\quad 20 / 21$ & BQll & NO & $7 \times 2$ (14 grupos) & fin de $2^{\underline{a}}$
\end{tabular}

Duplicación de grupos sin cambios en POD $\rightarrow$ Simplificación y adaptación de las prácticas

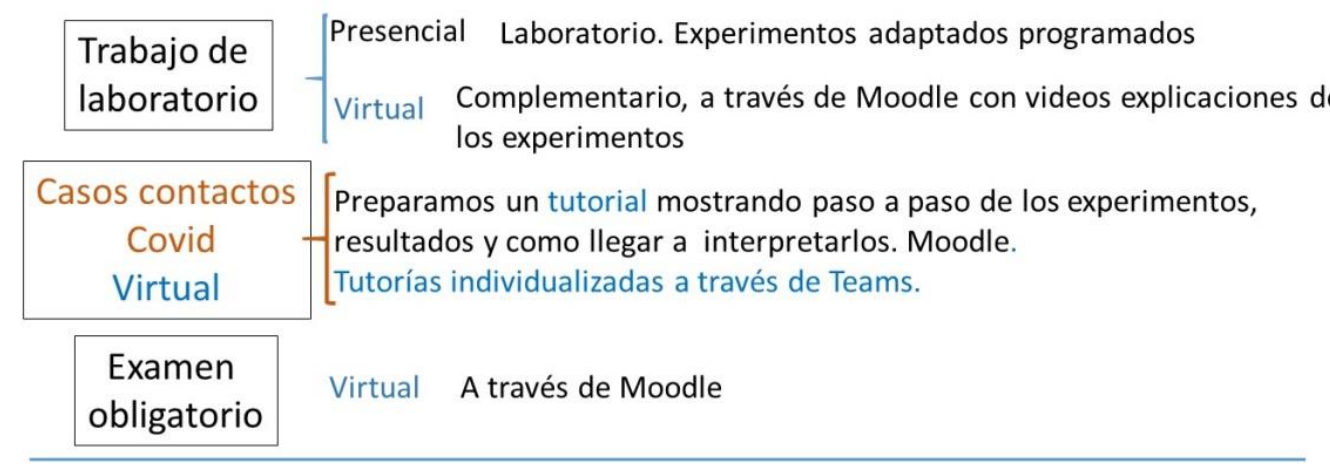

Tabla 3. Adaptaciones y circunstancias durante el desarrollo de la docencia práctica en la asignatura Bioquímica II (BQ-II). POD: Plan de Organización Docente. *Duplicación de grupos para reducir el número de personas por aula y cumplir con la "distancia Covid".

Durante el transcurso de las semanas de prácticas se dieron varios casos de alumnos que eran contactos estrechos de positivos en Covid19 y que por tanto perdieron varias sesiones de laboratorio. Para estos alumnos se preparó un tutorial de cada práctica con el paso a paso de los experimentos y la interpretación de los resultados. Además, las dudas se resolvieron con tutorías personalizadas por Teams.

Al finalizar la etapa de laboratorio, los estudiantes realizaron un examen de prácticas online programado en el Moodle. Los resultados alcanzados fueron similares a los de cursos anteriores. 


\begin{tabular}{|c|c|c|c|c|}
\hline \multirow[b]{2}{*}{ Período del curso } & \multirow{3}{*}{$\begin{array}{c}\text { Asignatura } \\
\text { BBM }\end{array}$} & \multicolumn{2}{|c|}{ Modalidad/grupos } & \multirow[b]{2}{*}{ Ola Covid19 } \\
\hline & & Virtual & Presencial* & \\
\hline 2o C $20 / 21$ & & 4 grupos $/ 7$ & $\rightarrow 3$ grupos $/ 7$ & 3 a \\
\hline
\end{tabular}

$\begin{gathered}\text { Trabajo con bases } \\ \text { de datos }\end{gathered}$
Presencial Aula de informática y conectados a Teams

\begin{tabular}{|c|c|c|}
\hline $\begin{array}{l}\text { Trabajo de } \\
\text { laboratorio }\end{array}$ & Virtual & $\begin{array}{l}\text { A través de Teams con videos explicaciones de los experimentos } \\
\text { y ejercicios }\end{array}$ \\
\hline & Presencial & Laboratorio realización de los experimentos programados \\
\hline $\begin{array}{c}\text { Tutorías } \\
\text { obligatorias }\end{array}$ & Virtual & $\begin{array}{l}\text { A través de Teams para debatir el trabajo derivado de la práctica } \\
\text { con bases de datos. }\end{array}$ \\
\hline
\end{tabular}

Tabla 4. Adaptaciones y circunstancias durante el desarrollo de la docencia práctica en la asignatura Bioquímica y Biología Molecular (BBM). *Duplicación de grupos para reducir el número de personas por aula y cumplir con la "distancia Covid".

En el segundo cuatrimestre del curso 2021 comenzamos la asignatura de tercer curso Bioquímica y Biología Molecular. En este caso estábamos hacia el final de la tercera ola. Como se muestra en la tabla 4. Las prácticas de esta asignatura se programaron en el tiempo y horas por prácticas considerando que podría darse el cambio a la docencia presencial durante el período programado marzo-abril 2021.

En la parte de trabajo con bases de datos se emplearon, como en años anteriores páginas web en las que se extraía información sobre un gen determinado y su regulación, con esta información los alumnos elaboraban un trabajo que incluía, además de la información encontrada, un modelo de regulación y un pequeño diseño experimental para probar una hipótesis planteada por ellos mismos. Esta parte se pudo desarrollar perfectamente de modo virtual. Para elaborar el trabajo por parejas, los estudiantes tenían que reunirse a través de Teams y posteriormente era obligatoria la asistencia a al menos una tutoría grupal a través de Teams. A menudo se dieron al menos dos reuniones/grupo con el profesorado. 
Las tutorías grupales para los trabajos de prácticas se concertaban previa cita y supusieron el aumento en la carga de trabajo del profesorado, no sólo por la preparación previa de materiales, sino que las tutorías grupales e individuales a través de Teams supusieron muchas horas de tele-docencia.

Las adaptaciones e innovaciones realizadas en nuestras asignaturas, exceptuando la clase invertida, incluyen las principales estrategias seguidas para clases de residentes de medicina en universidades americanas (Chick et al., 2020) en las que las teleconferencias, videos quirúrgicos y solución de cuestiones prácticas online fueron, al igual que en nuestra experiencia, una alternativa clave para superar la situación de pandemia.

\section{CONCLUSIONES}

Las principales reflexiones de esta experiencia son:

Conseguimos adaptar la docencia a través de Teams sin haber tenido experiencia previa. Superar este reto fue posible gracias al apoyo de la institución y de los compañeros de facultad.

También creamos y adaptamos materiales para la docencia por Moodle como tutoriales para seguir paso a paso cada experimento de prácticas de Bioquímica II.

Elaboramos nuevos videos con explicaciones por Teams. Este material servirá en todo o en parte para futuros cursos.

Conseguimos tutorías online eficientes para debatir los trabajos de prácticas y resolver dudas en el caso del examen de prácticas en BQ-II 0 en las tutorías para preparar trabajos de prácticas en BBM. El poder convertir a los alumnos en moderadores y que éstos compartan su escritorio y debatan sus ideas fue necesario también para la corrección de errores conceptuales. Los estudiantes superaron holgadamente las prácticas con los estándares de cursos anteriores. 
Cabe comentar que tanto alumnado, según expresaron los representantes en reuniones de coordinación, como el profesorado preferimos las prácticas presenciales. En relación a la opinión del alumnado, Chakraborty y colaboradores (2021) analizaron la opinión de estudiantes universitarios comparando la docencia presencial, a través de M00CS y online, destacando que ésta última fue la peor valorada. Desde otro punto de vista, también la consideraban estresante y estaba afectando a su vida social.

La interacción directa en el laboratorio con el profesorado y otros estudiantes permite no sólo el aprendizaje de las técnicas, sino que durante el desarrollo de los experimentos se puedan corregir defectos en la praxis que nunca se podrán detectar online además de favorecer la socialización entre iguales.

\section{REFERENCIAS}

Chakraborty, P., Mittal, P., Gupta, M.S., Yadav, S. \& Arora, A. (2021) Opinion of students on online education during the COVID-19 pandemic. Hum Behav \& Emerg Tech. 3, pp 357-365.

Chick, R. C., Clifton, G. T., Peace, K. M., Propper, B. W., Hale, D. F., Alseidi, A. A. \& Vreeland, T. J. (2020). Using Technology to Maintain the Education of Residents During the COVID-19 Pandemic. Journal of Surgical Education, 77(4), pp.729-732.

Nuere, S. \& De Miguel, L. (2020). The Digital/Technological Connection with COVID-19: An Unprecedented Challenge in University Teaching. Technology, Knowledge and Learning, pp.3-13. DOI: 10.1007/s10758-020-09454-6.

Picón, G. A., González Caballero, K. \& Paredes, N. (2020). Performance and educational training in digital competences in non-presential classes during the COVID-19 pandemic. SciElo Preprints. DOl: https://doi.org/10.1590/SciELOPreprints.778.

Zhu, X. \& Liu, J. (2020). Education in and After COVID-19: Immediate Responses and Long-Term Visions. Postdigital Science and Education, pp.1-5. D0l: 10.1007/s42438-020-00126-3. 